\title{
Evaluation of S2 alar and traditional S1 pedicle fixation for severe lumbar spondylolisthesis in different bone mineral densities: a finite element analysis
}

Juehan Wang

Sichuan University West China Hospital

Wei Chen

Sichuan University West China Hospital

Xi Yang

Sichuan University West China Hospital

Ce Zhu

Sichuan University West China Hospital

Tingxian Ling

Sichuan University West China Hospital

Ganjun Feng

Sichuan University West China Hospital

Yueming Song

Sichuan University West China Hospital

Limin Liu ( $\boldsymbol{\nabla}$ liulimin_spine@163.com )

Sichuan University West China Hospital

Research article

Keywords: Spondylolisthesis, Osteoporosis, S2 alar screw, Finite element analysis

Posted Date: December 28th, 2020

DOI: https://doi.org/10.21203/rs.3.rs-41056/v2

License: (c) (i) This work is licensed under a Creative Commons Attribution 4.0 International License. Read Full License 


\section{Abstract}

Purpose: Little is known about the biomechanical performance of L5-S2 alar internal fixation constructs after posterior lumbar interbody fusion. This study aimed to compare the biomechanical effect of L5-S1 internal fixation and L5-S2 alar internal fixation on severe lumbar spondylolisthesis.

Methods: A normal male volunteer without a history of spinal disease was selected, lumbar CT data was collected. An intact L5-S2 three-dimensional finite element model was created by Mimics and Geomagic. Two kinds of fixation methods were reconstructed including (1) the L5-S1 screw fixation model and (2) the L5-S2 alar fixation model. The inverse repair was performed using Geomagic software, the internal fixation device was drawn using Creo software, and the model parameters were set and analyzed using ANSYS Workbench software.

Results: The validity of the intact model shows that the ROM of the model is similar to that of a reported cadaveric study. The average stress of the L5-S2 alar internal fixation device was $86.9-111 \%$ higher(P区 0.001) than that of the L5-S1 fixation device when the bone of the S1 screw path reached the yield threshold. The maximal stress of the S1 screw in the L5-S2 alar fixation was significantly lower (P『0.001) than it in the L5-S1 fixation when the stress exceeds the S1 bone yield threshold. When the S1 screw path bone yielded, the maximal deformation value of the $S 1$ screw path was similar in both models $(P>0.05)$, while the average deformation value of the S1 screw path in L5-S2 alar fixation was significantly higher $(P \otimes 0.01)$ than it in L5-S1 fixation.

Conclusion: Extending fixation to the $\mathrm{S} 2$ wing can significantly improve internal fixation device stability and reduce the risk of intraoperative and postoperative fractures while avoiding injury to the sacroiliac joint, reducing the difficulty of surgery and the risk of injury to surrounding tissues. It is a reasonable plan for the treatment of moderate and severe lumbar spondylolisthesis with osteoporosis.

\section{Introduction}

Lumbar spondylolisthesis is a deformity that occurs between the lumbar vertebrae and the relative adjacent vertebrae, mainly consists of horizontal displacement, and is one of the most common spinal deformities. Wiltse et al. classified lumbar spondylolisthesis into six types based on the causative factors: dysplastic, isthmic, degenerative, traumatic, pathological and iatrogenic ${ }^{[1]}$. According to the Meyerding classification, when the vertebral displacement exceeds $50 \%$ of the adjacent lower vertebral body, moderate to severe slippage can be diagnosed. Severe lumbar spondylolisthesis may result in lumbar curvature changes, nerve compression, and decreased lumbar spine stability. Patients can present obvious symptoms of low back pain and often require surgical treatment. The main objectives of surgical treatment are to restore lumbar spine stability, relieve nerve strain and compression, and improve clinical symptoms.

In 1986, Matthiass proposed lever reduction using pedicle screws and a rod fixation system ${ }^{[2]}$. Since then, with the development of internal fixation materials and clinical practice, the application of pedicle screws 
in the treatment of lumbar spondylolisthesis has gradually become widespread. However, problems such as screw loosening, iatrogenic fracture and broken internal fixation devices have not been satisfactorily solved, especially screw loosening in osteoporotic patients. Severe osteoporosis is a significant cause of internal fixation failure, such as pedicle screw loosening and pull-out after spinal fusion surgery ${ }^{[3-5]}$. Spondylolisthesis often occurs in the L5/S1 segment because there is a high degree of mobility at the L4$\mathrm{S} 1$ segment, joining the rigid sacropelvic unit ${ }^{[6]}$. Failure of instrumentation frequently begins at the sacrum, which is the site of maximal stress ${ }^{[7]}$. The reported failure rate of $\mathrm{S} 1$ pedicle screws is approximately $44 \%{ }^{[8-9]}$. Thus, extension of the instrumentation to the distal sacrum or iliac wings has gained increasing interest. Alternatives to the single use of S1 screws include the addition of S2 alar screws and S2 alar-iliac screws.

Finite element analysis (FEA) is a numerical method simulating and analyzing the behavior or mechanism of structures or components, which is initially developed in the 1950s in the aircraft industry [10]. FEA has already been applied to characterize the complex biomechanical properties of the lumbar vertebrae in previous studies ${ }^{[11-15]}$. Nevertheless, to the best of our knowledge, few studies have illustrated the detailed biomechanical mechanisms of L5-S1 and L5-S2 alar fixation in spondylolisthesis patients with osteoporosis.

The aim of this study is to compare L5-S1 reduction and fixation methods and explore whether extending the fixation to include the $\mathrm{S} 2$ alar can significantly improve the stability of the internal fixation device by FEA.

\section{Materials And Methods}

\section{Construction of the intact model}

A 35-year-old healthy male volunteer with no history of lumbar disease was selected. We performed Computed tomography (CT) examination using a 160-row CT scanning system (Siemens Sensation 64, Siemens Medical Solutions, Forchheim, Germany). The scanning parameters were as follows: resolution: $1 \mathrm{~mm}$ resolution in-plane and $3 \mathrm{~mm}$ slice thickness; X-ray tube voltage and milliampere:120 kV and 200 mAs. The CT images were provided by the Department of Radiology at The West China Hospital, and stored in Digital Imaging and Communications in Medicine (DICOM) format. Informed written consent was obtained from the subject participating in the study.

The collected raw data were imported into Mimics Research 19.0 (Materialise NV, Leuven, Belgium) for three-dimensional (3D) reconstruction. Subsequently, the 3D model generated by Mimics was imported into Geomagic Studio 2013 (3D Systems, Inc., Rock Hill, South Carolina, USA) to simulate the transforaminal lumbar interbody fusion (TLIF) surgical procedure by the application of facetectomy, annulotomy, and soft tissue release for lumbar spondylolisthesis. Using the toggle Mask 3D Preview option, the editMask command was applied to remove the lower articular process on both sides of the L5 vertebra and the upper articular process on both sides of the S1 vertebra (Fig. 1). The spikes and features 
were deleted, smoothing was performed with a polygon mesh, and the triangles were made more uniform in size. Then, a patch was generated using the following tools: Construct Patches and Grid and Fit Surfaces. The smoothed model was saved and imported into ANSYS Workbench 19.0 (ANSYS, Ltd., Canonsburg, Pennsylvania, USA). Each vertebra was modeled as consisting of a cancellous inner core surrounded by a $0.5 \mathrm{~mm}$ cortical shell. A $1 \mathrm{~mm}$ bony end plate was simulated on either end of each vertebra (Fig. 2) ${ }^{[13]}$. The material properties of the various spinal components were derived from the literature ${ }^{[16-19]}$, as specified in Table 1.

\begin{tabular}{lcccc}
\hline Component/Materials & $\begin{array}{c}\text { Density } \\
\square \mathrm{kg} / \mathrm{m}^{3} \square\end{array}$ & $\begin{array}{c}\text { Young Modulus } \\
(\mathrm{MPa})\end{array}$ & $\begin{array}{c}\text { Poisson } \\
\text { Ratio }\end{array}$ & $\begin{array}{c}\text { yield stress } \\
\square \mathrm{MPa} \square\end{array}$ \\
\hline Cancellous bone & $160 / 240 / 320$ & $57 / 143 / 267$ & 0.2 & $2.2 / 3.9 / 5.9$ \\
Cortical bone & 1910 & 12000 & 0.3 & 100 \\
$\begin{array}{l}\text { Spinal } \\
\text { instrumentation } \\
\text { (titanium alloy) }\end{array}$ & 4430 & 110000 & 0.3 & 860 \\
\hline
\end{tabular}

Table 1. Material Properties Used in the Finite Element Model

\section{Modeling of implants}

The posterior instrumentation consisted of transpedicular screws and longitudinal rods spanning between adjacent screws and was modeled by Creo Parametric 6.0 computer-aided design (CAD) (PTC, Boston, MA, USA). According to the size of the patient's vertebral body,the L5 and S1 pedicle screws were modeled as $45 \mathrm{~mm}$ in length and $6 \mathrm{~mm}$ in diameter, and the S2 alar pedicle screws were modeled as 60 $\mathrm{mm}$ in length and $6 \mathrm{~mm}$ in diameter. Titanium material properties were applied for the posterior instrumentation.

\section{Construction of models with two different fixation options and different bone mineral densities}

The screws and rods were assembled with the lumbar spine model to construct the two models separately in ANSYS Workbench 19.0 (ANSYS, Ltd., Canonsburg, Pennsylvania, USA). Repeating the above simulated screw placement and subsequent steps, by fine-tuning the pedicle screw angle and depth, a total of 72 models were built, including 36 cases of L5-S1 fixation and 36 cases of L5-S2 alar fixation, and the bone mineral density (BMD) in each fixation model was divided into low, medium and high ${ }^{[20]}$. The specific BMD values are shown in Table 1.

\section{Contact definitions}


A finite sliding algorithm with a coefficient of friction of 0.2 was defined between the pedicle screws and screw paths to allow for any small relative displacements. The pedicle screws were placed such that they engaged approximately two-thirds of the vertebral body.

\section{Loading and boundary conditions}

A motion protocol was defined for all reconstructive options and the intact lumbar spine condition. Both sides of the sacroiliac joint surface were immobilized throughout the load simulation. A sustained $150 \mathrm{~N}$ preload parallel to the L5 upper endplate was imposed on the bilateral L5 pedicle screws to simulate the intraoperative pull-out strength when the spondylolisthesis was reduced (Fig. 3). Screws were judged to have started to loosen when the average stress of the screw path bone was close to the yield stress. The

yield threshold of the S1 screw path, the stress distribution of the two different internal fixation methods and the $\mathrm{S} 1$ bone deformation were analyzed and compared to investigate the biomechanical properties of L5-S1 fixation and L5-S2 alar fixation.

\section{Statistical analysis}

Data were analyzed using SPSS 19.0 and are represented as the mean \pm SD. Analysis of variance (ANOVA) or Student's t test was performed to measure the statistical significance of differences, and $\mathrm{P}<$ 0.05 was considered statistically significant.

\section{Results}

\section{Model validation}

We assessed the range-of-motion (ROM) of the intact model under different physiological motions, including flexion, extension, left lateral bending, right lateral bending, and rotation with $5 \mathrm{Nm}$ and $10 \mathrm{Nm}$ of moment respectively. The rotation angles obtained were compared with experimental data found in the literature. Figure 4 presents the rotation angles for this study and the rotation angles obtained by the mentioned authors, for $10 \mathrm{Nm}$ and $5 \mathrm{Nm}$, respectively. Our Obtained angles are consistent with the results found in the relevant literature ${ }^{\text {[21] }}$

\section{Maximal stresses of the $\mathrm{S} 1$ screw path}

In the L5-S1 fixation model, the maximal von Mises stresses were found on the upper contact surface at the tail of the screw path. Nevertheless, in the L5-S2 alar model, the maximal von Mises stresses were found at the caudal lateral side of the screw path (Fig. 4). Both in high, medium and low BMD models, when the bone of the S1 screw path reached the yield threshold (5.9 MPa, 3.9 MPa, 2.2 MPa), the corresponding stresses in the L5-S2 alar model were $86.9 \%-111 \%$ higher than that of L5-S1 model, which meant L5-S2 alar fixation could withstand greater stress and gain more stability. A comparative analysis 
of the relevant data is shown in Table 2, and the corresponding relationship between the load and BMD is shown in Fig. 5.

\begin{tabular}{cccccc}
\hline Group & Cases & Low-BMD & Medium-BMD & High-BMD & $p$ \\
\hline L5-S1 & 36 & $355.58 \pm 11.5$ & $593.17 \pm 19.6$ & $803.42 \pm 24.2$ & $\square 0.001$ \\
L5-S2alar & 36 & $664.75 \pm 9.2$ & $1148.25 \pm 16.0$ & $1695 \pm 23.4$ & $\square 0.001$ \\
\hline$p$ & & $\square 0.001$ & $\square 0.001$ & $\square 0.001$ & \\
increasing rate & & $86.9 \%$ & $93.6 \%$ & $111 \%$ &
\end{tabular}

Table 2: Comparison of the yield threshold of the S1 screw path in the two groups of models $\square \mathbf{x}$ $\pm S \square N \square$

\section{Stress of internal fixation}

The internal fixation stress distribution of the L5-S1 model and the L5-S2 alar model is shown in Figure 3. In the L5-S1 model, the maximal stress was concentrated at the tail of the screw, while in the L5-S2 alar model, the maximal stress was concentrated on the connecting rod near the S1 screw (Fig. 6). Both in high, medium and low BMD models, when the stress of S1 bone reached yield threshold, the maximal stress of L5-S1 model was much higher than that in the L5-S2 alar model, Which indicated the L5-S1 fixation method may undergo higher stress and was more prone to internal fixation failure. A statistical comparative analysis is shown in Table 3.

\begin{tabular}{cccccc}
\hline Group & Cases & Low-BMD & Medium-BMD & High-BMD & $p$ \\
\hline L5-S1 & 36 & $423.25 \pm 9.0$ & $689.83 \pm 14.569$ & $945.33 \pm 20.155$ & $\square 0.001$ \\
L5-S2 alar & 36 & $225.42 \pm 9.1$ & $373.00 \pm 15.0$ & $537.58 \pm 21.865$ & $\square 0.001$ \\
\hline
\end{tabular}
$p$
$\square 0.001$
$\square 0.001$
$\square 0.001$

Table 3: Comparison of the maximal stress of the S1 screw in the two groups of models when the stress exceeds the $S 1$ bone yield threshold $\square x^{-} \pm S \square M P a \square$

\section{S1 screw path deformation}

In the L5-S1 model, the most obvious deformation of the S1 path was on the upper contact surface of the tail, followed by the lower contact surface of the anterior segment of the screw path. The deformation of the middle and rear screw path was the least, and the internal and external deformation was similar. In the L5-S2 alar model, the largest deformation was located at the tail of the screw path, and the overall distribution was relatively uniform (Fig. 7). Regardless of BMD, when the S1 screw path bone yielded, the maximum deformation value of the $S 1$ screw path was similar in both models $(P>0.05)$, while the average deformation value of the $\mathrm{S} 1$ screw path was significant different (P凶0.01), as shown in Tables 4 and 5. 


\begin{tabular}{ccccc}
\hline Group & Cases & Low-BMD & Medium-BMD & High-BMD \\
\hline L5-S1 & 36 & $0.15 \pm 0.02$ & $0.13 \pm 0.15$ & $0.12 \pm 0.13$ \\
L5-S2 alar & 36 & $0.24 \pm 0.01$ & $0.24 \pm 0.01$ & $0.24 \pm 0.01$ \\
\hline$p$ & & $\square 0.001$ & $\square 0.001$ & $\square 0.001$ \\
\hline
\end{tabular}

Table 4: Comparison of mean values of S1 bone deformation in the two groups when S1 screw path bone yielded $\llbracket \bar{x}^{-} \pm s \rrbracket m m \square$

\begin{tabular}{ccccc}
\hline Group & Cases & Low-BMD & Medium-BMD & High-BMD \\
\hline L5-S1 & 36 & $0.48 \pm 0.05$ & $0.43 \pm 0.05$ & $0.42 \pm 0.04$ \\
L5-S2 alar & 36 & $0.46 \pm 0.02$ & $0.43 \pm 0.02$ & $0.41 \pm 0.02$ \\
\hline$p$ & & $\square 0.05$ & $\square 0.05$ & $\square 0.05$
\end{tabular}

Table 5: Comparison of maximum values of 1 bone deformation in the two groups when the S1 screws began to loosen $\square \bar{x}^{-} \pm S \square \mathrm{mm} \square$

\section{Discussion}

In the current surgical treatment of lumbar spondylolisthesis, L5-S1 internal fixation is mostly reported for reduction; this method has been widely used for many years with proven efficacy, but it also has shortcomings ${ }^{[21]}$. Due to the short force arm and relatively concentrated stress, this L5-S1 reduction method based on the lever principle is prone to S1 screw loosening in patients with moderate to severe spondylolisthesis, especially in the case of osteoporosis ${ }^{[22]}$. In response to this problem, surgical approaches continue to be explored and modified, including extended internal fixation to S2, sacroiliac joint fixation and iliac fixation ${ }^{[23]}$. From a biomechanical point of view, extended methods must be able to increase the stability of the internal fixation device and provide greater power for lifting and reduction. However, each extended internal fixation method still has some flaws. The use of sacroiliac screw fixation inevitably leads to sacroiliac joint damage, loss of sacroiliac joint mobility, and increased long-term risk of chronic sacroiliac pain ${ }^{[24]}$. Due to the anatomy of the $\mathrm{S} 2$ pedicle, which is relatively short and close to the anterior internal iliac artery, extended fixation to the $\mathrm{S} 2$ pedicle may lead to insufficient holding power, limited auxiliary ability and even possibly massive hemorrhage ${ }^{[23]}$. The iliac screw fixation device has a complex structure and increased the risk of local soft tissue injury, chronic pain, and even skin breakdown. These complications are more frequently reported in older patients with osteoporosis ${ }^{\text {[25-27] }}$. To the best of our knowledge, there is no study that have examined the biomechanical differences between L5-S1 and L5-S2 alar fixation in severe spondylolisthesis with different BMDs.

This study presents a preliminary biomechanical evaluation of L5-S1 and L5-S2 alar fixation with different BMDs to analyze the intraoperative reduction stress distribution in severe lumbar spondylolisthesis patients with osteoporosis via FEA. Regarding the yield threshold of the S1 screw path, 
in the L5-S1 fixation method, when lifting and repositioning the site of lumbar spine slippage, the stress was concentrated on the tail of the S1 screw path, which was in accordance with previously reported data [28]. Because of the thinner cortex of the sacrum compared to that of other vertebrae, excessive concentrated stress could lead to screw loosening or even internal fixation failure. This may also explain why screw loosening often occurs in the tail segment. Nevertheless, in the L5-S2 alar fixation method, the $\mathrm{S} 1$ screw path stress was uniformly distributed. When the bone in the S1 screw path yielded, the average stress of the L5-S2 alar model was $86.9 \%-111 \%$ higher than that of the L5-S1 model, which could significantly improve the reduction ability and S1 screw stability, especially in the low BMD group. This result could be observed more visually in the reduction load-BMD diagram (Fig. 5). When the BMD and the load required for reduction were below the "L5-S1" line, fixation with both 4 screws and 6 screws could achieve reliable stability. However, when the BMD and the load required for reduction were between the "L5-S1" and the "L5-S2 alar" lines, the L5-S1 fixation method could lead to internal fixation failure or could not provide good sagittal alignment, while the L5-S2 alar method could provide reliable reduction. This result can be of value in informing clinical practice. For instance, in a spondylolisthesis patient with severe osteoporosis treated in our department, ideal reduction was not achieved after L5-S1 fixation because of the inadequate lifting force, and compared to another patient with the same conditions, extended fixation to the S2 alar could obviously help him/her achieve better postoperative sagittal alignment (Fig. 8).

Regarding the stress of internal fixation, analysis of the $\mathrm{S} 1$ internal fixation stress in both models revealed that the $\mathrm{S} 1$ screw stress was concentrated at the tail of the screw in both groups and distributed on the upper and lower contact surfaces, while the L5-S2 alar model showed relatively dispersed S1 screw stress. The results indicated that during reduction, compression of the screw against the bone mainly occured on the upper contact surface of the caudal segment of the screw path. Screw cutting and pull-out in the superoposterior direction could occur when the bone yield threshold was reached. When subjected to the same reduction load, the stress of the S1 screw tail in the L5-S1 model was much higher than that in the L5-S2 alar model, predicting a higher risk of screw bending or even fracture. In the L5-S2 alar fixation model, the maximal stress was found on the connecting rod near the $\mathrm{S} 1 \mathrm{screw}$, reaching 319$942 \mathrm{MPa}$ in different BMDs. However, it was still lower than the maximal stress (423-945 MPa) of the S1 screw in the L5-S1 model at the same density. Therefore, the following can be concluded: 1 . at the same BMD, the L5-S2 alar model had a higher risk of titanium rod deformation or even fracture, while the L5-S1 model had a higher risk of screw fracture; and 2. when the $S 1$ bone yield stress was reached, the risk of titanium rod deformation in the L5-S2 alar model was still lower than the risk of screw fracture in the L5S1 model, which further proved that the overall structure of the L5-S2 alar model was more stable.

Regarding S1 screw path deformation, as the reduction load increased, there were different degrees of bone deformation in both models, mainly concentrated in the anterior and caudal segments of the S1 screw path, with downward compression deformation in the anterior segment and upward compression deformation in the caudal segment. The deformation of the screw path meant that compression fracture occurred in the S1 bone, and the range of fracture increased with increasing reduction load. When the S1 
bone yield stress was reached, the average S1 screw path deformation in the L5-S2 alar model was $60 \%$ to $100 \%$ higher than that in the L5-S1 model, while there was no significant difference in the maximum screw path deformation between the two models. The above results indicated the following: 1 . with the same reduction effect (i.e., the same reduction load was applied) and the same BMD, in the L5-S2 alar model, the deformation of the S1 screw path was dispersed and uniform, while in the L5-S1 model, local bone destruction at the front and tail ends of the screw path easily occurred due to stress concentration; 2. When the $S 1$ bone reached yield threshold, the degree of $S 1$ screw path deformation had little correlation with bone density, according to Table 4; and 3. S1 screw loosening could occur with relatively minor bone destruction in the L5-S1 model, whereas it occurred with more bone deformation in the L5-S2 alar model, indirectly proving that the L5-S2 alar model was more stable.

Unlike in the large majority of available finite element studies, we decided not to analyze only a group of single representative models but to construct a total of 72 models by fine-tuning the angle and depth of the screw to stimulate intraoperative errors. Although previous studies have reported the biomechanics

of sacropelvic fixation ${ }^{[29,30]}$, we first further discussed the advantages of the L5-S2 alar fixation model in detail in severe spondylolisthesis patients with osteoporosis by setting different BMDs compared to the L5-S1 fixation.

This study has some limitations. This model is only representative of a partial population and cannot fully reflect the lumbosacral conditions at different ages. Furthermore, to simplify the computations, only three overall BMD levels were set instead of reconstructing the lumbosacral bone according to the CT gray values. Moreover, the model did not consider the influence of the various muscle tissues and ligaments and thus cannot reflect the true in vivo conditions.

\section{Conclusion}

L5-S2 alar fixation can withstand a greater reduction load and has a relatively lower risk of rod or screw fracture. In summary, compared with L5-S1 fixation, extended fixation to the S2 alar has better stability and potential for improved reduction outcomes, especially in severe lumbar spondylolisthesis patients with severe osteoporosis.

\section{Abbreviations}

CT: Computed tomography; FEA: Finite element analysis; BMD: Bone mineral density; TLIF: Transforaminal lumbar interbody fusion; ANOVA: Analysis of variance.

\section{Declarations}

\section{Authors' contributions}

All contributing authors have read and approved the manuscript in its present form. JHW - data collection, study design, data analysis and manuscript writing. WC - data collection, study design, data 
analysis and manuscript writing. $\mathrm{XY}$ - performing surgeries, study design. $\mathrm{CZ}$ - data collection, data analysis. TXL-data analysis. GJF - performing surgeries, supervising rehabilitation. YMS - performing surgeries, study supervisor, performing surgeries, study design and manuscript review. LML - study supervisor, performing surgeries, study design and manuscript review.

\section{Acknowledgements}

Not applicable.

\section{Funding}

This study was supported in part by the Projects of the Science \& Technology Department of Sichuan Province: NO.2017SZ0046 and NO.2017SZDZX0021.

\section{Availability of data and materials}

The datasets used and/or analyzed during the current study are available from the corresponding author on reasonable request.

\section{Ethics approval and consent to participate}

This study was approved by the Ethics Committee of the West China Hospital. The participant consent was written and was performed in accordance with the ethical standards of the Declaration of Helsinki.

\section{Consent for publication}

Not applicable.

\section{Competing interests}

All authors declare that they have no competing interests

\section{References}

[1] Wiltse LL, Newman PH, Macnab I. Classification of spondylolisis and spondylolisthesis. Clin Orthop Relat Res. 1976;(117):23-29.

[2] Matthiass HH, Heine J. The surgical reduction of spondylolisthesis. Clin Orthop Relat Res. 1986; (203):34-44.

[3] Esses SI, Sachs BL, Dreyzin V. Complications associated with the technique of pedicle screw fixation. A selected survey of ABS members. Spine (Phila Pa 1976). 1993;18(15):2231-2239. doi:10.1097/00007632-199311000-00015 
[4] Reinhold M, Schwieger K, Goldhahn J, Linke B, Knop C, Blauth M. Influence of screw positioning in a new anterior spine fixator on implant loosening in osteoporotic vertebrae. Spine (Phila Pa 1976). 2006;31(4):406-413. doi:10.1097/01.brs.0000199894.63450.70

[5] Krishnan V, Varghese V, Kumar GS. Comparative Analysis of Effect of Density, Insertion Angle and Reinsertion on Pull-Out Strength of Single and Two Pedicle Screw Constructs Using Synthetic Bone Model. Asian Spine J. 2016;10(3):414-421. doi:10.4184/asj.2016.10.3.414

[6] Eismont FJ, Norton RP, Hirsch BP. Surgical management of lumbar degenerative spondylolisthesis. J Am Acad Orthop Surg. 2014;22(4):203-213. doi:10.5435/JAAOS-22-04-203

[7] Birknes JK, White AP, Albert TJ, Shaffrey Cl, Harrop JS. Adult degenerative scoliosis: a review. Neurosurgery. 2008;63(3 Suppl):94-103. doi:10.1227/01.NEU.0000325485.49323.B2

[8] Emami A, Deviren V, Berven S, Smith JA, Hu SS, Bradford DS. Outcome and complications of long fusions to the sacrum in adult spine deformity: luque-galveston, combined iliac and sacral screws, and sacral fixation. Spine (Phila Pa 1976). 2002;27(7):776-786. doi:10.1097/00007632-200204010-00017

[9] Orita S, Ohtori S, Eguchi Y, et al. Radiographic evaluation of monocortical versus tricortical purchase approaches in lumbosacral fixation with sacral pedicle screws: a prospective study of ninety consecutive patients. Spine (Phila Pa 1976). 2010;35(22):E1230-E1237. doi:10.1097/BRS.0b013e3181e5092c

[10] Hu BW, Lv X, Chen SF, Shao ZW. Application of Finite Element Analysis for Investigation of Intervertebral Disc Degeneration: from Laboratory to Clinic. Curr Med Sci. 2019;39(1):7-15. doi:10.1007/s11596-019-1993-7

[11] Lu T, Lu Y. Comparison of biomechanical performance among posterolateral fusion and transforaminal, extreme, and oblique lumbar interbody fusion: a finite element analysis. World Neurosurg. 2019;129:e890-e899.

[12] Chung SK, Kim YE, Wang KC. Biomechanical effect of constraint in lumbar total disc replacement: a study with finite element analysis[J]. Spine, 2009, 34(12):1281-1286.

[13] Zhou QK, Zeng FH, Tu JL, Dong ZQ, Ding ZH. Influence of cement-augmented pedicle screw instrumentation in an osteoporotic lumbosacral spine over the adjacent segments: a 3D finite element study. J Orthop Surg Res. 2020;15(1):132. Published 2020 Apr 7. doi:10.1186/s13018-020-01650-5

[14] Du CF, Cai XY, Gui W, et al. Does oblique lumbar interbody fusion promote adjacent degeneration in degenerative disc disease: A finite element analysis [published online ahead of print, 2020 Nov 21]. Comput Biol Med. 2020;128:104122. doi:10.1016/j.compbiomed.2020.104122

[15] Wang Md K, Jiang PhD C, Wang PhD L, Wang Md H, Niu PhD W. The biomechanical influence of anterior vertebral body osteophytes on the lumbar spine: A finite element study. Spine J. 2018;18(12):2288-2296. doi:10.1016/j.spinee.2018.07.001 
[16] Cho W, Wang W, Bucklen B, Ramos RG, Yassari R. The Role of Biological Fusion and Anterior Column Support in a Long Lumbopelvic Spinal Fixation and Its Effect on the S1 Screw - An In-Silico Biomechanics Analysis [published online ahead of print, 2020 Nov 4]. Spine (Phila Pa 1976).

2020;10.1097/BRS.0000000000003768. doi:10.1097/BRS.0000000000003768

[17] Fidalgo DS, Areias B, Sousa LC, et al. Minimally invasive transforaminal and anterior lumbar interbody fusion surgery at level L5-S1. Comput Methods Biomech Biomed Engin. 2020;23(8):384-395. doi:10.1080/10255842.2020.1731482

[18] Areias B, Caetano SC, Sousa LC, et al. Numerical simulation of lateral and transforaminal lumbar interbody fusion, two minimally invasive surgical approaches. Comput Methods Biomech Biomed Engin. 2020;23(8):408-421. doi:10.1080/10255842.2020.1734579

[19] Wang B, Hua W, Ke W, et al. Biomechanical Evaluation of Transforaminal Lumbar Interbody Fusion and Oblique Lumbar Interbody Fusion on the Adjacent Segment: A Finite Element Analysis. World Neurosurg. 2019;126:e819-e824. doi:10.1016/j.wneu.2019.02.164

[20] Zhang QH, Tan SH, Chou SM. Effects of bone materials on the screw pull-out strength in human spine. Med Eng Phys. 2006;28(8):795-801. doi:10.1016/j.medengphy.2005.11.009

[21] Tropiano P, Giorgi H, Faure A, Blondel B. Surgical techniques for lumbo-sacral fusion. Orthop Traumatol Surg Res. 2017;103(1S):S151-S159. doi:10.1016/j.otsr.2016.06.023

[22] Kashlan ON, Chen KS, La Marca F. Lumbosacral and Pelvic Fixation Techniques[M]. In: Essentials of Spinal Stabilization. Holly LT, Anderson PA (editors). Cham: Springer International Publishing; 2017. pp. 401-412.

[23] Koller H, Zenner J, Hempfing A, Ferraris L, Meier O. Reinforcement of lumbosacral instrumentation using S1-pedicle screws combined with S2-alar screws. Oper Orthop Traumatol. 2013;25(3):294-314. doi:10.1007/s00064-012-0160-0

[24] Lee SH, Jin W, Kim KT, Suk KS, Lee JH, Seo GW. Trajectory of transsacral iliac screw for lumbopelvic fixation: a 3-dimensional computed tomography study. J Spinal Disord Tech. 2011;24(3):151-156. doi:10.1097/BSD.0b013e3181e7c120

[25] Chang TL, Sponseller PD, Kebaish KM, Fishman EK. Low profile pelvic fixation: anatomic parameters for sacral alar-iliac fixation versus traditional iliac fixation. Spine (Phila Pa 1976). 2009;34(5):436-440. doi:10.1097/BRS.0b013e318194128c

[26] Emami A, Deviren V, Berven S, Smith JA, Hu SS, Bradford DS. Outcome and complications of long fusions to the sacrum in adult spine deformity: luque-galveston, combined iliac and sacral screws, and sacral fixation. Spine (Phila Pa 1976). 2002;27(7):776-786. doi:10.1097/00007632-200204010-00017 
[27] Kim JH, Horton W, Hamasaki T, Freedman B, Whitesides TE Jr, Hutton WC. Spinal instrumentation for sacral-pelvic fixation: a biomechanical comparison between constructs ending with either S2 bicortical, bitriangulated screws or iliac screws. J Spinal Disord Tech. 2010;23(8):506-512. doi:10.1097/BSD.0b013e3181c37438

[28] Chatzistergos PE, Magnissalis EA, Kourkoulis SK. A parametric study of cylindrical pedicle screw design implications on the pullout performance using an experimentally validated finite-element model. Med Eng Phys. 2010;32(2):145-154. doi:10.1016/j.medengphy.2009.11.003.

[29] Galbusera F, Casaroli G, Chande R, et al. Biomechanics of sacropelvic fixation: a comprehensive finite element comparison of three techniques. Eur Spine J. 2020;29(2):295-305. doi:10.1007/s00586-01906225-5

[30] Casaroli G, Galbusera F, Chande R, et al. Evaluation of iliac screw, S2 alar-iliac screw and laterally placed triangular titanium implants for sacropelvic fixation in combination with posterior lumbar instrumentation: a finite element study. Eur Spine J. 2019;28(7):1724-1732. doi:10.1007/s00586-01906006-0

\section{Figures}

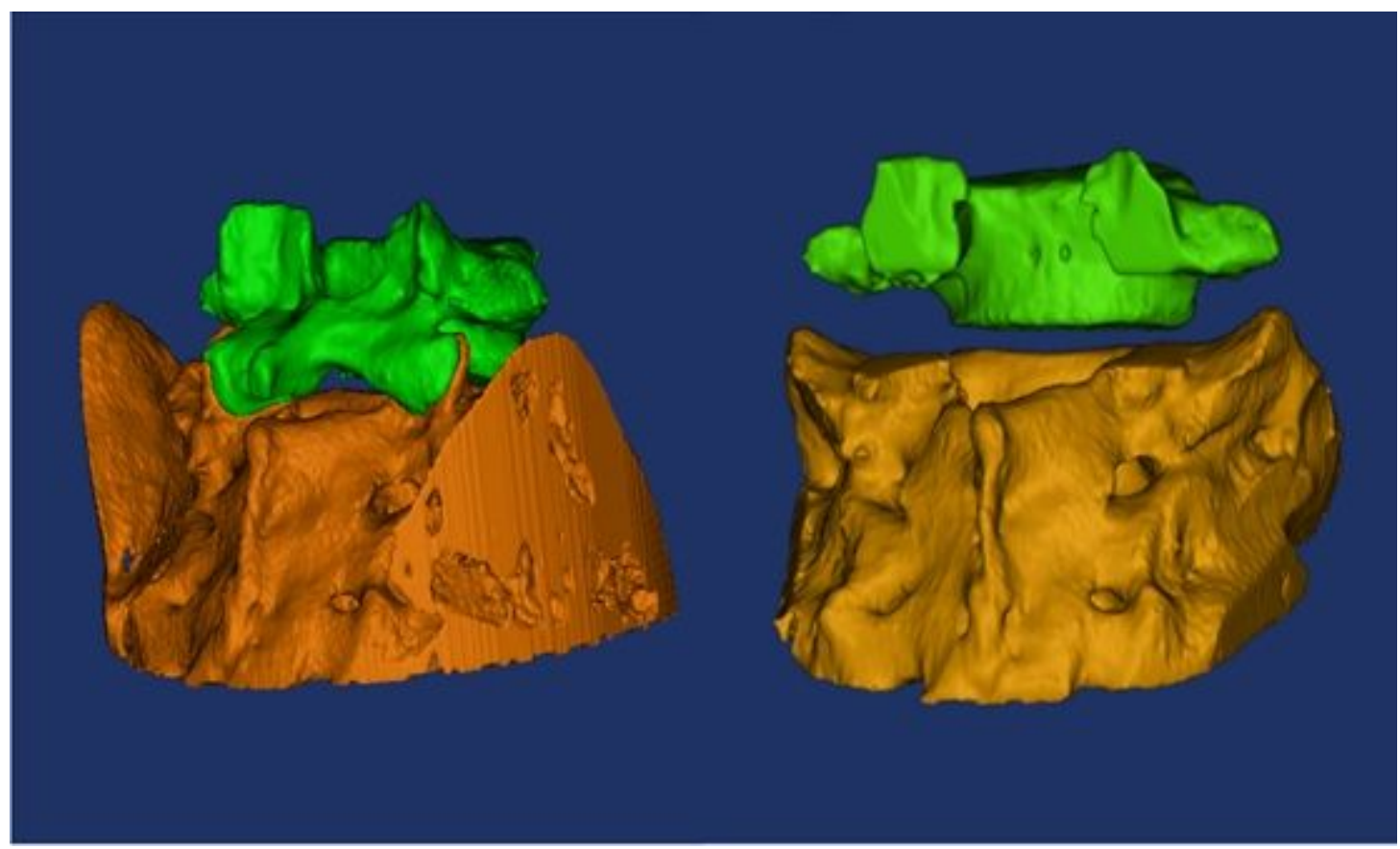

\section{Figure 1}

The original 3D lumbosacral model (left) and the final 3D lumbosacral model after smoothing and editing. 


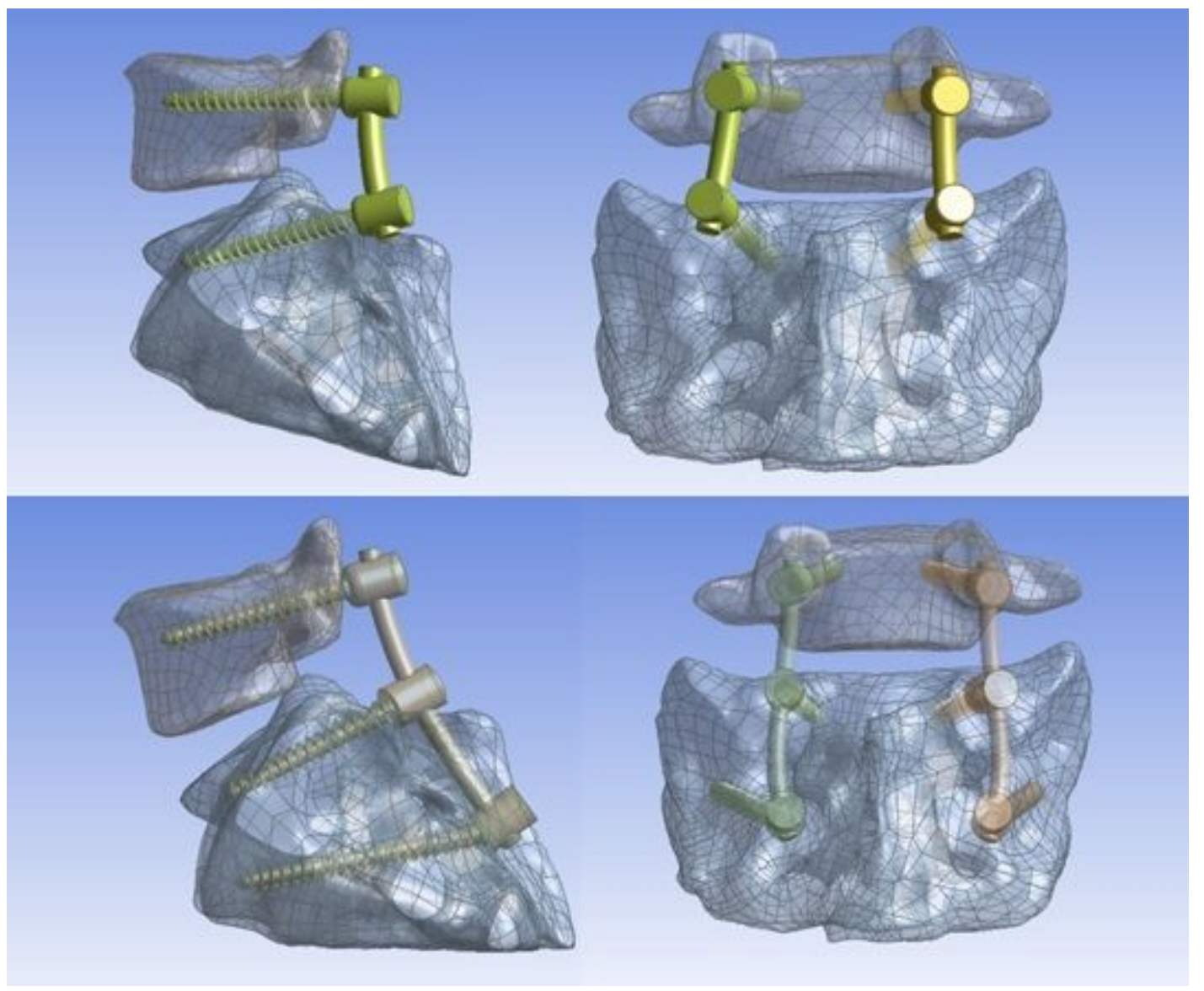

\section{Figure 2}

Lateral (left panel) and posterior (right panel) views of the lumbosacral configurations investigated.

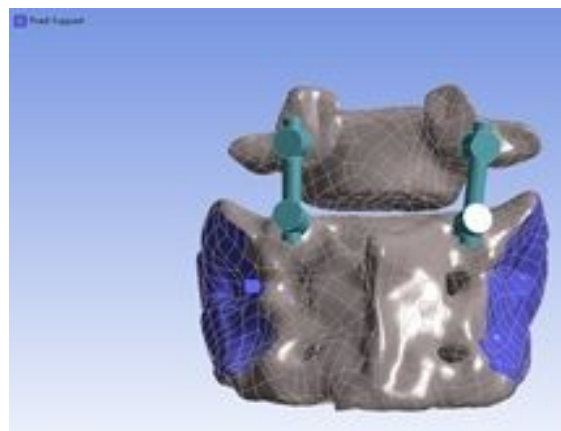

(a)

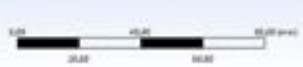

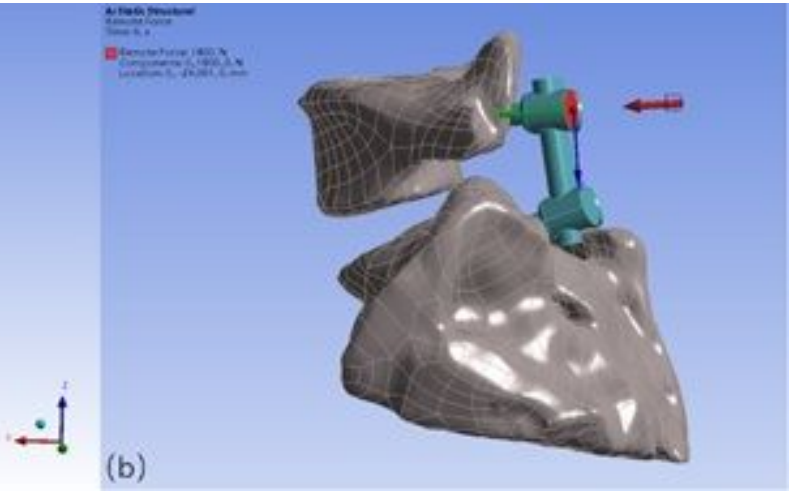

(b)

\section{Figure 3}

arrow represents the preload employed parallel to the : Loading and boundary conditions set for the lumbosacral configurations investigated: (a) boundary conditions of the intact model (the purple area represents the immobilized sacroiliac joint surfaces); (b) loading conditions (the red L5 upper endplate). 
(a)

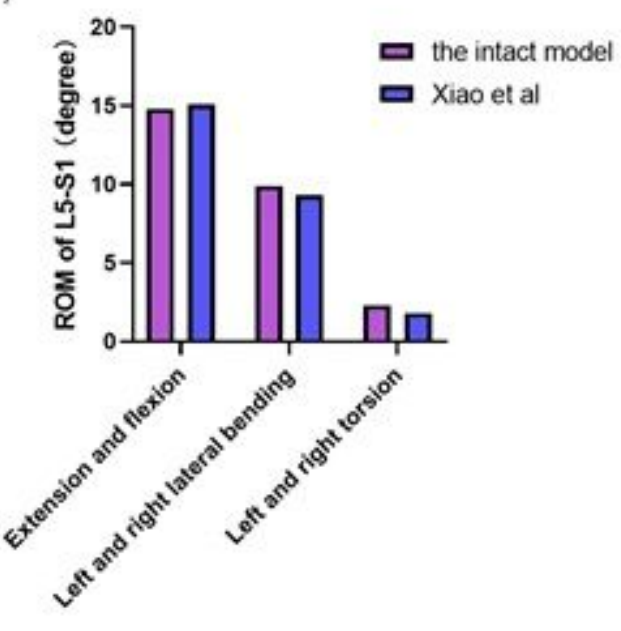

(b)

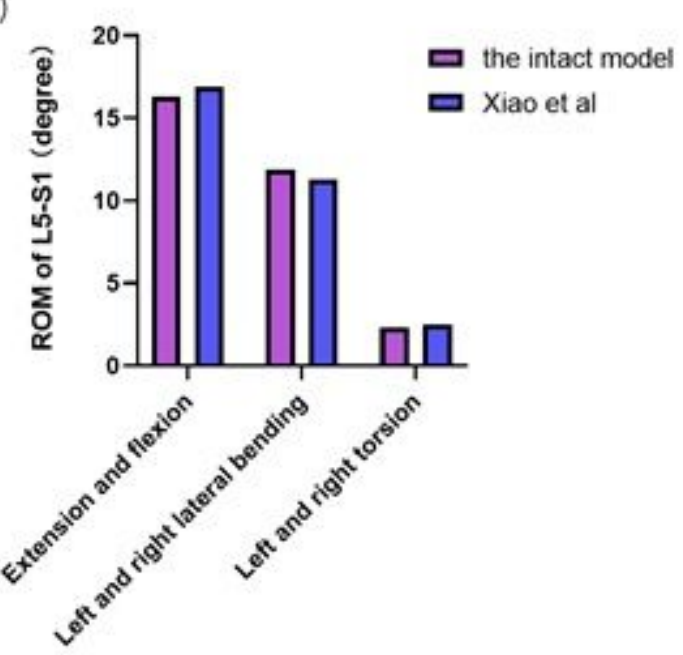

\section{Figure 4}

Model validation:comparation with previous reported results between different rotation angles for loads of $5 \mathrm{Nm}$ (a) and $10 \mathrm{Nm}$ (b).

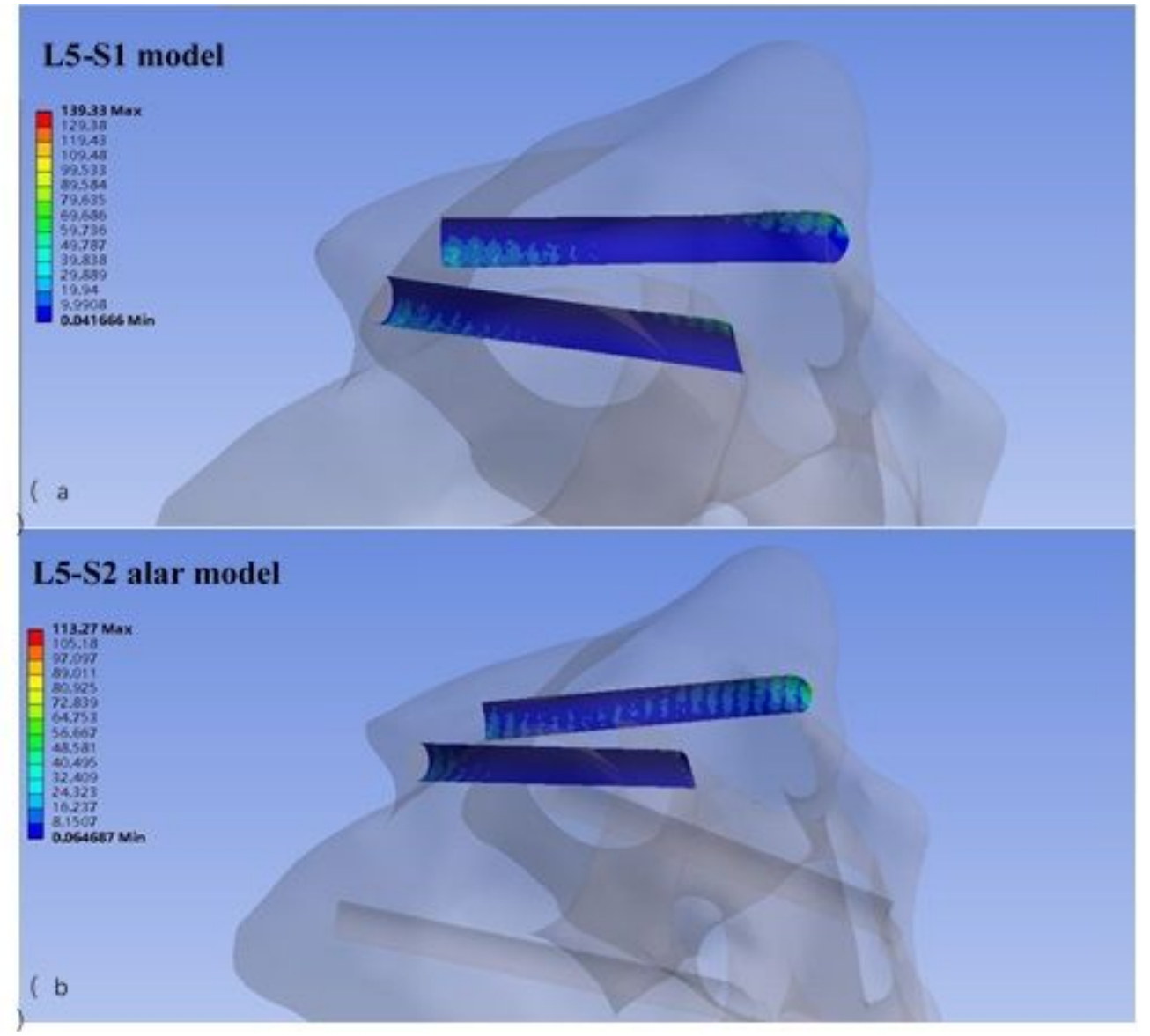

Figure 5

Stress distribution of the S1 screw path. The figure above shows the L5-S1 model(a) and the figure below shows the L5-S2alar model(b) 


\section{Reduction load (N) $-\mathrm{BMD}\left(\mathrm{kg} / \mathrm{m}^{3}\right)$}

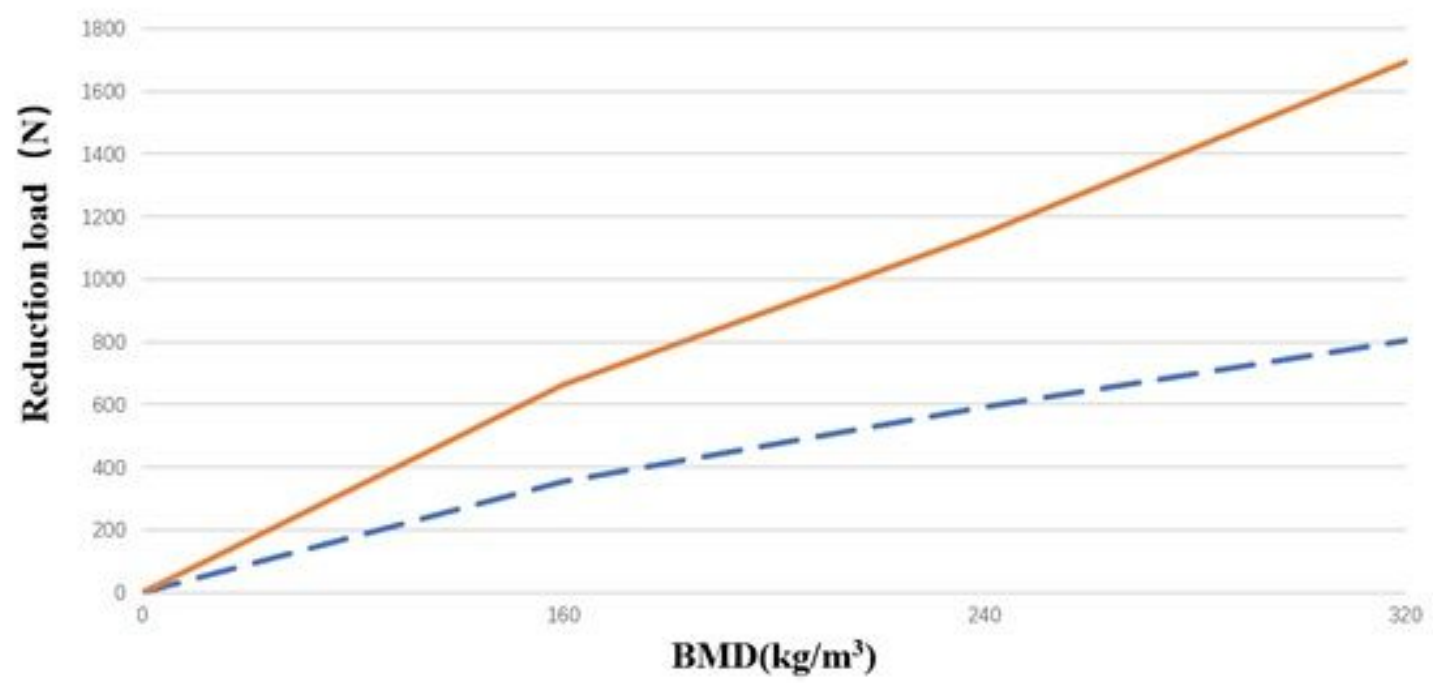

- $\cdot 15$-\$1

\section{Figure 6}

Correspondence between the reduction load and BMD. In the range below the load-BMD curve of the L5S1 model, the corresponding reduction load was relatively low, and even L5-S1 fixation can achieve reliable fixation. Within the range between the "L5-S1" curve and the "L5-S2 alar" curve, the required reduction load was moderate, and L5-S1 fixation could not provide good stability; therefore, the L5-S2 alar fixation method should be selected. In the range above the "L5-S2 alar" curve, the required load was too high, and even L5-S2 alar fixation could not provide reliable stability. 


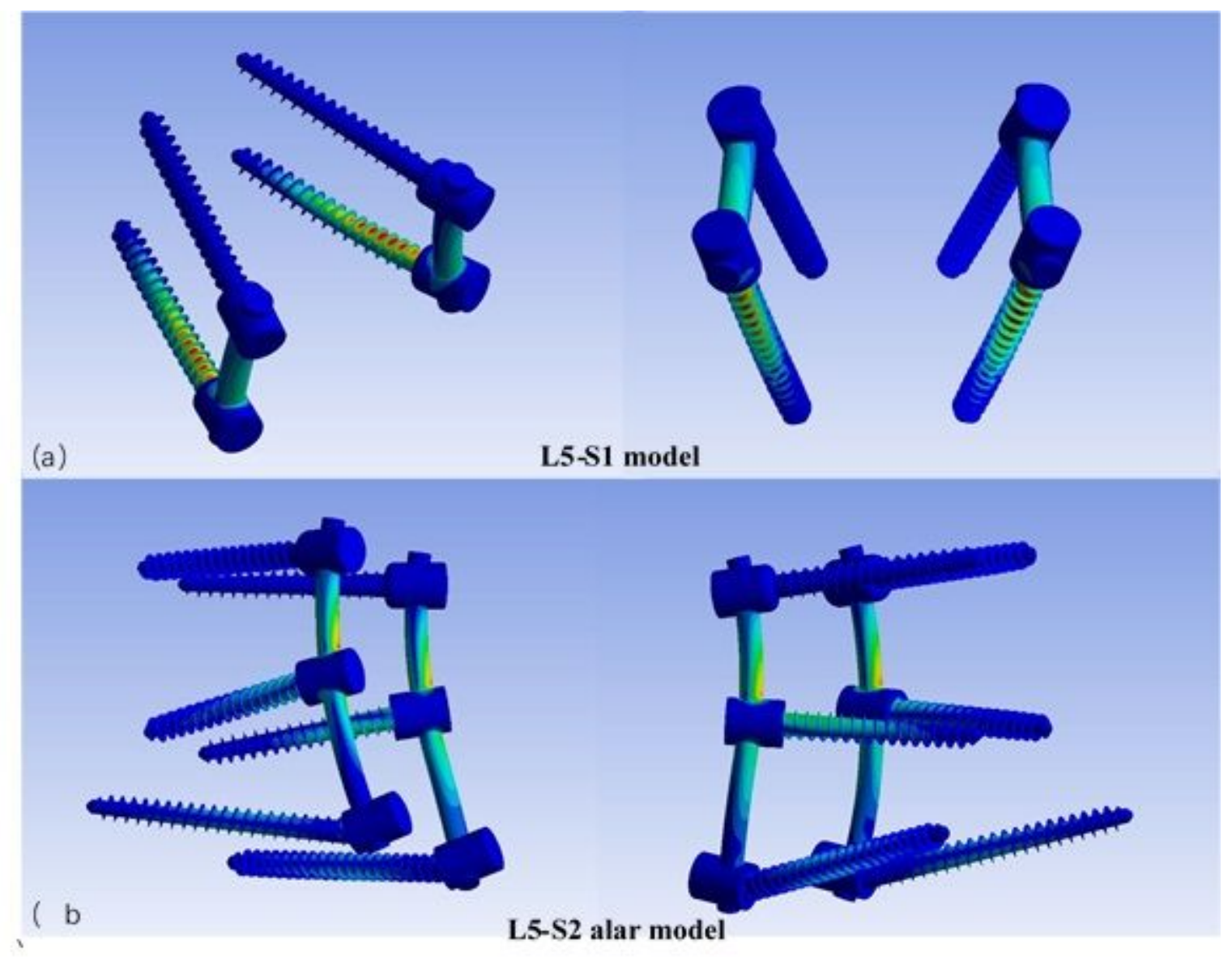

\section{Figure 7}

Stress distribution of internal fixation in the two models. The figure above shows the L5-S1 model(a) and the figure below shows the L5-S2alar model(b). 


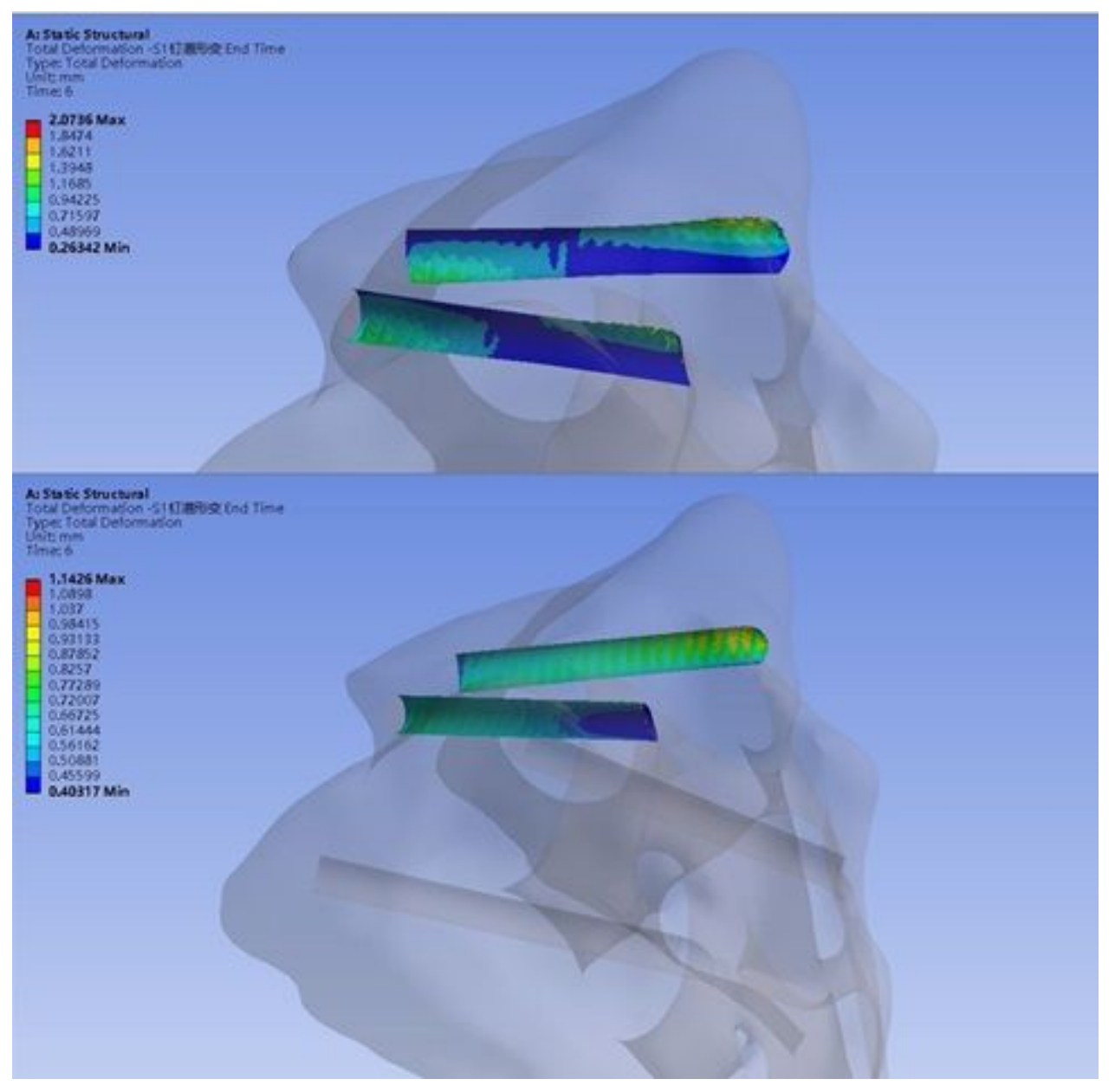

\section{Figure 8}

Deformation distribution of the $\mathrm{S} 1$ screw path in the two groups of models under low BMD conditions. The figure above shows the L5-S1 model and the figure below shows the L5-S2alar model. 

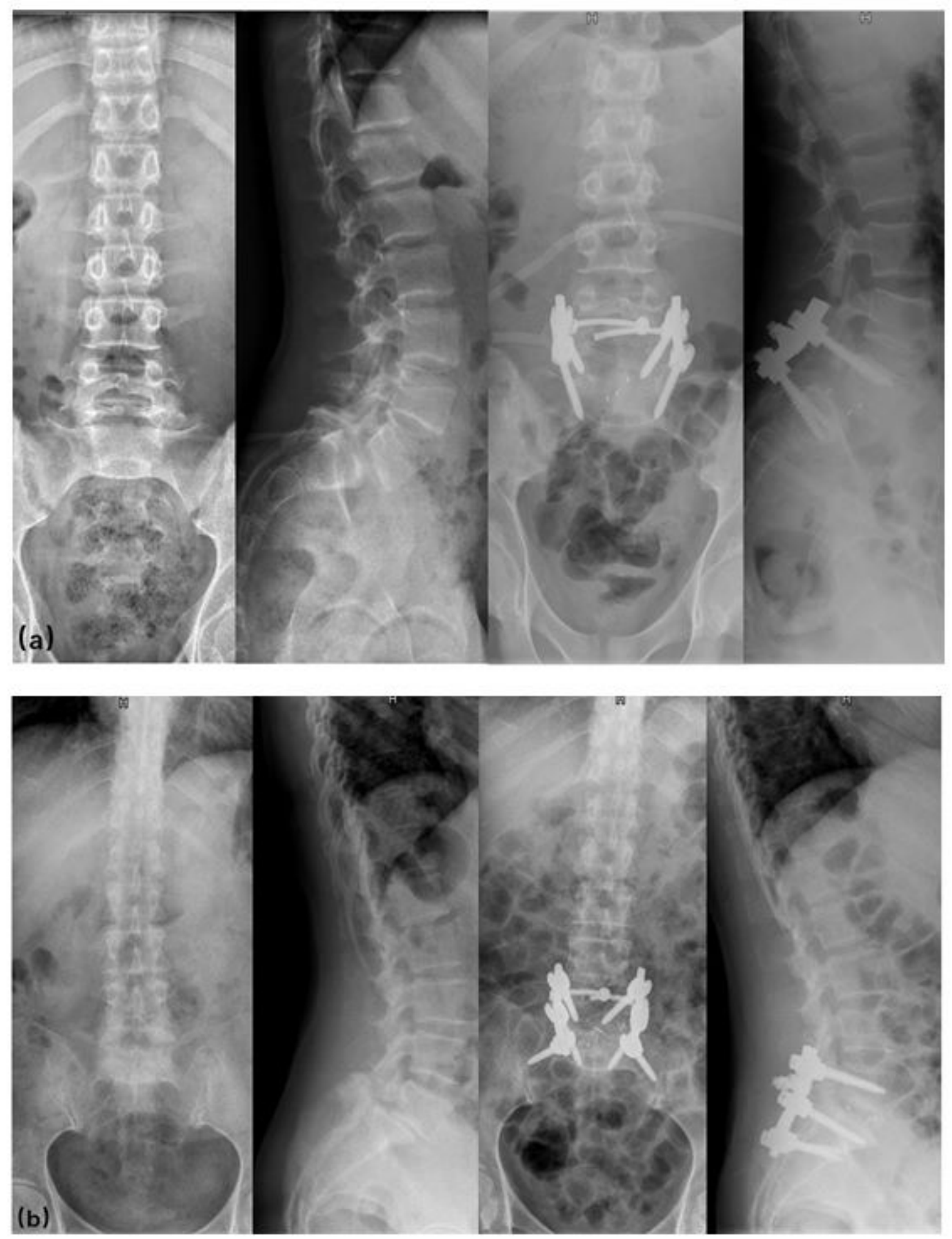

Figure 9

(a) Preoperative and postoperative X-rays of spondylolisthesis patient treated with L5-S1 fixation; (b) Preoperative and postoperative X-rays of spondylolisthesis patient treated with L5-S2 alar fixation. 